Canadian



Botany

Botanique

\title{
Quantitative developmental analysis of two phenotypes of Hibiscus rosa-sinensis in the context of Homeosis
}

\begin{tabular}{|r|l|}
\hline Journal: & Botany \\
\hline Manuscript ID & cjb-2017-0185.R1 \\
\hline Manuscript Type: & Article \\
\hline Date Submitted by the Author: & 03-Feb-2018 \\
\hline $\begin{array}{r}\text { Complete List of Authors: } \\
\text { No Montréal, sciences biologiques } \\
\text { Lacroix, Christian; University of Prince Edward Island, Biology }\end{array}$ \\
\hline $\begin{array}{r}\text { Is the invited manuscript for } \\
\text { consideration in a Special } \\
\text { Issue? : }\end{array}$ & N/A \\
\hline Keyword: & logistic regression, ontogeny, spatial constraints, floral meristem, homeosis \\
\hline &
\end{tabular}


Title: Quantitative developmental analysis of two phenotypes of Hibiscus rosa-sinensis in the context of Homeosis

Christian H. Norton ${ }^{1}$ and Christian R. Lacroix*

Department of Biology, The University of Prince Edward Island, 550 University Avenue, Charlottetown, PE, C1A 4P3, Canada

* Corresponding author: Christian R. Lacroix, Department of Biology, University of Prince Edward Island, 550 University Avenue, Charlottetown, PE, C1A 4P3, Canada

Tel.: 902-620-5231

E-mail: lacroix@upei.ca

Keywords: logistic regression, scanning electron microscopy, ontogeny, spatial constraints, floral meristem, homeosis, double flower

${ }^{1}$ Current institutional affiliation: Institut de recherche en biologie végétale (Université de Montréal), 4101 Sherbrooke Est, Montréal, QC, H1X 2B2, Canada (christian.norton@umontreal.ca) 


\begin{abstract}
The flowers of Hibiscus rosa-sinensis L. (Malvaceae) exist in two floral morphologies: a single phenotype and a double phenotype. This study focused on the early stages of floral development, just before the initiation of petal primordia and up until the bifurcation of the stamen primordia. The two phenotypes were compared using logistic regression and bootstrapping techniques. Four aspects of floral development were considered: (a) organogenesis of petal and stamen primordia, and stamen bifurcation, (b) stamen primordia allometry (c) stamen primordia morphology and (d) stamen primordia size. The single and double buds initiated petal primordia at the same bud radii, but double buds initiated stamen primordia and stamen bifurcation at larger bud radii than the single phenotype. Double stamen primordia were shorter, wider, and more spherical than single stamen primordia, although the sizes of the single and double stamen primordia (defined as the sum of their length and width measurements) were not different. Results suggest that the additional space on the floral meristem of the double phenotype is linked to the divergent development of stamen primordia occupying this extra space.
\end{abstract}




\section{Introduction}

Double-ness is well studied as a floral trait due to its relevance to plant breeding. Double flowers, with their fuller appearance, are considered by most to be more attractive than the simpler single flowers (Nakatsuka et al. 2015), and double flowers may also have greater market value for some cultivated plants (Scovel et al. 1998; Freyre et al. 2012). Research into breeding flowers of a double phenotype in Petunia hybrida showed that double-ness has inheritance probabilities like traits that are controlled by several genes, highlighting multiple genetic differences between the single and double phenotypes (Liu et al. 2016). Research into doubleness in poppy, tobacco, carnation, and potentilla also suggest that double-ness is a trait controlled by a combination of dominate and recessive alleles (Belyaeva 1995; Scovel et al. 1998: Zainol 1998;), in addition to environmental stressors such as temperature and moisture (Roberston 1984; Innes et al. 1989). It seems that the causes of double-ness is very case dependent, but there is considerable consensus that differences in the expression of C-class organ identity genes are a strong predictor of double-ness in ornamental flowers (Kapoor et al. 2005; Dubois et al. 2010; Galimba et al. 2012; Sun et al. 2014; Nakatsuka et al. 2015). The molecular mechanisms serve as context in which organogenesis operates. Quantitative analysis can inform what may be happening at the molecular level concerning the establishment of organ identity.

Hibiscus rosa-sinensis belongs to the Malvaceae (mallow) family; it is a eudicotyledonous, tropical to sub-tropical plant (MacIntyre and Lacroix 1996). Hibiscus rosasinensis exists in two distinct floral morphologies: single phenotype and double phenotype (Fig. 1). The double flowers exhibit merged petal-stamen structures called petaloids that fall under the continuum based-description of homeosis in plants described previously (Sattler 1988; MacIntyre and Lacroix 1996). The single flowers consist of five, single-lobed petals with stamens projecting laterally from the distal end of the staminal tube (Fig. 1 images A and C); there is a clear 
distinction between petals and stamens in the single flowers. The double flowers do not have a clear distinction between petals and stamens; the double flowers have a much fuller appearance due to the blended petal-stamen structures found in abundance between the clearly defined petals and stamens (Fig.1 images B and D). These merged petal-stamen structures exhibit both petal and stamen morphological characteristics to varying extents.

Previous work with $H$. rosa-sinensis sought to determine at what stage during floral development a morphological difference could be observed between doubles and singles (MacIntyre and Lacroix 1996). This earlier work looked at the initiation of petal and stamen primordia on the five-lobed, floral meristematic ring. Results from this work suggested that singles and doubles could be distinguished at the earliest point in development based on patterns of organ initiation and size. Unlike singles, doubles do not maintain any discernable pattern of organ initiation beyond the initial petal primordia. Furthermore, organ primordia of doubles are not all of roughly equal size, compared to singles (MacIntyre and Lacroix 1996). However, this previous study did not mathematically quantify the distinction between singles and doubles in the early stages of floral development.

Many studies have used comparative contexts to characterize divergent organ development to great success. In Begonia varieties, a comparative context and allometry were used to characterize early leaf development as a means to understand phylogeny (McLellan 1990). McLellan (1990) found that varieties had different paths of morphological development that led to the same leaf morphology. Cohen et al. (2012) compared homostylous and heterostylous plant species in the Lithospermum genus to put developmental differences in the context of an evolutionary history. Nighiloo and Classen-Blockhoff (2016) used a comparative ontological context to understand differences in the number of floral structures per floral organ whorl in two closely related genera of the tribe Rubieae. In the case of $H$. rosa-sinensis, a 
comparative morphological study is relevant because it helps us understand the early sequence of organogenetic events that lead to divergent morphological pathways that correspond to the single and double phenotypes. In the case of the double phenotype, the identity of stamen primordia (be it more petal-like or more stamen-like) is not immediately obvious and only becomes defined as development progresses. Again, turning to Nighiloo and Classen-Blockhoff (2016), we agree that, "ontogenetic studies add a temporal component to [our] knowledge of mature structures." We may understand the genetic underpinnings that lead to phenotypic differences, and we may discern obvious morphological differences between fully developed flowers of different phenotypes, but we lack an understanding of the morphological pathways that define divergent development in H. rosa-sinensis. In short, we aim to supplement our current knowledge of the molecular mechanism that leads to floral homeosis with the complementary morphological mechanism that also leads to floral homeosis.

This paper focuses on the stamen primordia because MacIntyre and Lacroix (1996) noted morphological and developmental differences between the stamen primordia of the single and double phenotypes, and their results suggested that these unusual petal-anther structures develop from what otherwise seem to be typical stamen primordia. In this study we use a quantitative approach to analyze and compare the early floral development of the single and double phenotypes. The single and double phenotypes will be compared using four developmental criteria: (a) organogenesis of petal primordia, stamen primordia, and stamen primordia bifurcation (b) stamen primordia allometry (c) stamen primordia morphology and (d) stamen primordia size. The goal of this research is to understand the morphological changes during floral development that lead to double-ness and thus homeosis in H. rosa-sinensis. 


\section{Materials and Methods}

\section{Specimen Preparation}

Six doubles of the same phenotype and six singles of the same phenotype of $H$. rosasinensis were ordered from Avon Valley Floral Nursery in Dartmouth, Nova Scotia: “Golden Sunrise" was the single phenotype and "Mango Mist" was the double phenotype. Plants were kept in a greenhouse during the spring and summer, and in climate-controlled growth chambers during the fall and winter. Growth chambers were maintained at $27^{\circ} \mathrm{C}, 80 \%$ humidity, and twelve-hour cycles of light and darkness. The light intensity in the growth chamber ranged from 14710 lux to 15080 lux.

Shoot-tips were collected and stored in an FAA fixative consisting of 5:5:90 by volume $38 \%$ percent formaldehyde, glacial acetic acid, and 70\% ethanol. Collected shoot tips were stored in FAA for at least 24 hours to ensure specimens were infiltrated by the fixative. After fixation, flower buds on shoot tips were dissected to collect the floral primordia. In total, 30 single buds and 52 double buds were successfully collected from the shoot tips, all at various stages of development. Dissected buds were stored in FAA until all collected shoot tips were dissected.

Scanning Electron Microscopy (SEM)

Upon completion of the dissections, specimens were prepared for critical point drying (CPD). Specimens were first transferred from FAA to $70 \%$ ethanol. Specimens went through an alcohol dehydration series ending in 100\% ethanol: each stage of the alcohol dehydration series was at least 24 hours in duration. Once in 100\% ethanol, specimens were dried using a Quorum K850 critical point dryer. Once dried, specimens were mounted on SEM stubs using an adhesive sticker. The base of the specimen was painted with silver paint to increase the conductance between the mounted specimens and the stub. Mounted and painted specimens were then sputter 
coated with gold and palladium to a thickness of approximately 300 angstroms using a Denton Vacuum Desk II sputter coater. Finally, coated specimens were digitally imaged using a Hitachi TM3000 SEM and imaging software associated with the SEM. In total, 30 buds of the single phenotype and 52 buds of the double phenotype were imaged. With respect to measuring stamen primordia, 16 of the single buds and 18 of the double buds had measurable stamen primordia.

\section{Measurements}

Measurements of stamen primordia were taken from top views of floral buds during a specific, early frame of floral development: after the initiation of the stamen primordia and before their bifurcation. Ten stamen primordia were selected from each bud. Primordia were selected using a spiral pattern that began by choosing a stamen primordium furthest from the centre of the bud and then measuring additional primordia while moving clockwise and towards the centre of the bud. In the case of some of the less developed buds, there were less than ten measurable stamen primordia and efforts were made to measure as close to ten primordia as possible. The length (L) and width (W) of the selected ten primordia were measured using integrated imaging software associated with the Hitachi TM3000 SEM. By convention, length was set as the longer measurement. Length and width measurements were taken perpendicular to each other. In addition to length and width three radial measurements were taken for each bud and averaged $\left(\mathrm{R}_{\mathrm{av}}\right)$. An average radius was used to compensate for buds not being level on the stub, thus making it difficult to measure the radius once and be confident that the measure was accurate.

These measurements were used to approximate relative age (or stage of development), shape, and size of the buds and stamen primordia. The average radius of the bud acted as an approximation for developmental progression; the larger the radius, the more developed the bud was likely to be. The shape of stamen primordia was calculated by dividing the length of a 
stamen primordium by its width $(\mathrm{L} / \mathrm{W})$ to give the shape ratio. A higher shape ratio indicated a more elliptical stamen primordium. The size of a stamen primordium was defined as the sum of its length and width measurements. .

\section{Organogenesis}

Logistic regression was used to quantify and compare the initiation and early stages of development of stamen primordia between the single and double phenotypes. In preparation for logistic regression, each bud had to be visually inspected and assigned a value of 0 or 1 with respect to the presence or absence of petal primordia, stamen primordia, and stamen primordia bifurcation. As an example, the logistic regression analysis for petal primordia required visually assessing each photo of the single and double buds for evidence of petal primordia initiation. Buds without petal primordia were assigned a value of 0 , and buds with petal primordia were assigned a value of 1 . This process was repeated individually for stamen primordia and stamen primordia bifurcation, both cases using visual inspection to discern evidence of stamen primordia and stamen primordia bifurcation. There are fewer buds in the stamen bifurcation analysis because only buds that already had stamen primordia were deemed appropriate to include in this part of the analysis.

For all statistical analyses, R v.0.99.903 was used. A logistic regression model using Firth's penalized-likelihood method was fit for petal initiation, stamen initiation, and stamen bifurcation in both the single and the double flowers. Firth's method for logistic regression was used to address data separation in the organ presence vs. absence data (Firth 1993; Heinz and Schemper 2002). From each logistic regression model, an inflection point was calculated by rearranging the equation of the model. The inflection point is the value of the predictor at which the probability of the modelled outcome happening vs. not happening is $50 \%$. In this case, the 
inflection point is the average floral radius $\left(\mathrm{R}_{\mathrm{av}}\right)$ at which the probability of having the specified organ primordia vs. not having the specified organ primordia is $50 \%$. Bootstrapping $(\mathrm{R}=10000)$ was used to estimate $95 \%$ confidence intervals around each inflection point. Inflection points with bootstrapped 95\% confidence intervals were plotted for each developmental event in both the single and double phenotypes. The significance of each logistic regression model was checked using the likelihood ratio test, a chi-square statistic.

In addition to estimating 95\% confidence intervals, bootstrapped distributions of inflection points were also used to determine if the radii at which developmental events occurred as estimated by logistic regression were significantly different between the single and double floral forms. Bootstrapped distributions of inflection points for each developmental event in the single and double flowers were compared using a Welch's ANOVA, and a post-hoc pairwise comparison was run using the Dunnett-Tukey-Kramer pairwise multiple comparison test adjusted for unequal variances and unequal sample sizes.

\section{Stamen primordia allometry}

An allometric relationship is defined by the exponential equation $Y=a X^{m}$, where " $a$ " is a constant and " $m$ " is the relative rate of growth between $Y$ and $X$, i.e. the allometry. By putting the equation in its linear logarithmic form, we get $\ln (Y)=\ln (a)+(m) \ln (X)$. In this from, " $m$ " is the slope of line describing the relationship between $\ln (Y)$ and $\ln (X)$. Linear regressions on logged measurements, and the resulting linear equations, give us the ability to understand how stamen primordia length, width, and floral meristem radius scale with each other by comparing values of "m."

Linear regressions were performed on the natural logarithms of stamen primordia length vs. floral meristem radius, stamen primordia width vs. floral meristem radius, and stamen 
primordia width vs. floral meristem length for both the single and double phenotype. The significance of each linear regression was tested using an F-test. The slope values from the equations describing each linear regression were used to understand the relative differences in growth rates between (a) the morphological measurements being compared and (b) between phenotypes. If the value of the slope of the linear logarithmic form was less than one, the morphological measurement on the $\mathrm{x}$-axis was increasing in magnitude more quickly than the morphological measurement on the y-axis. A slope of one indicates that the magnitude of the $\mathrm{x}$ and y-axes are increasing proportionally to each other, and a slope of greater than one means that the $\mathrm{y}$-axis is increasing in magnitude more quickly than the $\mathrm{x}$-axis.

\section{Stamen primordia morphology and size}

In the following analysis, only comparisons that produced a significant $\mathrm{p}$-value from the permutation T-tests were considered viable candidates for a logistic regression and bootstrapping. For the following logistic regression models, the single buds were assigned the value of 0 , and the double buds were assigned the value of 1 . The significance of each logistic regression model was checked using the likelihood ratio test. The likelihood ratio test is a chi-square statistic.

Permutation T-tests $(R=10000)$ were used to compare the length, width, and shape ratio of stamen primordia between single and double flowers. Standard logistic regressions were used to predict the inflection points for each morphological measurement. In this case, the inflection point is the length, width, or shape at which a stamen primordium was as equally likely to be from a single as from double floral bud. Bootstrapped distributions of inflection points were then used to estimate $95 \%$ confidence intervals around each inflection point for stamen primordia length, width, and shape. 
Permutation T-tests $(R=10000)$ were also used to compare the stamen primordia size between the single and double phenotypes. Standard logistic regressions were used to predict the inflection points for size. In this case, the inflection point is the size at which a stamen primordium was as equally likely to be from a single as from double floral bud. Bootstrapped distributions of inflection points were then used to estimate $95 \%$ confidence intervals around the size inflection point.

\section{Results}

\section{Visual Assessment of Developmental Morphology}

The floral development of the single (Fig. 2) and double (Fig. 3) phenotypes shared the same general stages of early development. First, a spherical floral ring meristem developed from which the organ primordia would be initiated (Fig. 2, images A and B; Fig. 3, images A and B). Next, the spherical meristem became increasingly pentagonal in shape as five distinct areas became clear and a circular depression began forming in the middle of the meristem (Fig. 2, image C; Fig. 3, image C). Petal primordia were initiated on the edge of the ring shortly after the floral meristem took on this pentagonal morphology with the circular depression. Following the initiation of petal primordia on the edge of the ring of meristematic tissue, stamen primordia were initiated on the surface of the ring of meristematic tissue, first becoming apparent at the edge of the circular depression in the centre of the floral meristem (Fig. 2, images C and D; Fig. 3, images D and E). Stamen primordia were initiated in five groups, each group made up of two rows of stamen primordia running radially from the centre of the ring of the floral meristem. The initiation of stamen primordia was followed by the bifurcation of stamen primordia along their minor axis (Fig. 2, images $\mathrm{G}$ and H; Fig. 3, image H). After the initiation of stamen primordia, 
the development of the single and double phenotypes appeared to diverge most drastically with respect to stamen primordia organization, organogenesis, and structure.

In the context of the developmental frame that was studied, petal primordia of single buds were wider with less distance between each of the petal primordia (Fig. 2, images F, G, and H) than petal primordia of double buds which were longer at the end of the developmental frame, and had more space between them (Fig. 3, images G and H). Petal primordia overlapped in the single buds only.

The single and double buds showed differences with respect to the symmetry and organization of stamen primordia initiation. Stamen primordia on the single buds were initiated in five, well-ordered groups, each groups made up of two parallel lines of stamen primordia running radially from the centre to the edge of the bud (Fig. 2, images C, D, and E). Stamen primordia on the double buds were also initiated in five groups, but the groups lacked the same degree of uniformity of shape, size, and order that was seen in the stamen primordia from the single buds (Fig. 3, images D, E, and F).

Concerning bud topography, single buds were relatively flat throughout the developmental frame of interest (Fig. 2, images E, F, G, and H) whereas double buds started out flatter (Fig. 3, images C and D) and then became more cone-like in appearance towards the end of the developmental frame of interest (Fig. 3, images G and H).

\section{Organogenesis: Petal Primordia}

The photos of single and double buds in Figures 2 and 3 described in the previous section represent individual buds at specific stages of development. The quantitative analyses that follow from here take into consideration all the samples we measured. The quantitative results should 
not be compared against the individual buds in Figures 2 and 3 that are only used to illustrate the general development of the single and double phenotypes.

The initiation of petal primordia on single buds took place at an average bud radius of $190.9 \mu \mathrm{m}$ (Fig. 4). For every $1 \mu \mathrm{m}$ increase in bud radius, a single bud was $8.2 \%$ more likely to have petal primordia (likelihood ratio test $=28.60, p<0.001$ ). Double buds initiated petal primordia at an average bud radius of $192.0 \mu \mathrm{m}$ (Fig. 4). For every $1 \mu \mathrm{m}$ increase in bud radius, a double bud was $10.8 \%$ more likely to have petal primordia (likelihood ratio test $=56.11, p<$ 0.001). The average bud radius for petal primordia initiation was not significantly different between the single and double phenotypes, suggesting both phenotypes produced petal primordia at about the same size (Fig. 4, $p>0.05$ ).

\section{Organogenesis: Stamen Primordia and Stamen Bifurcation}

With respect to the initiation and bifurcation of stamen primordia, buds of the single phenotype showed evidence of these developmental events at smaller bud radii than the buds of the double phenotype (Fig. 4). The difference between single and double flowers for radii was statistically significant at the stage of initiation of stamen primordia and stamen bifurcation (Fig. 4). The initiation of stamen primordia on single buds occurred at a bud radius of $202.5 \mu \mathrm{m}$ and stamen bifurcation at $302.6 \mu \mathrm{m}$ (Fig. 4). For every $1 \mu \mathrm{m}$ increase in bud radius, a single bud was $7.8 \%$ more likely to have stamen primordia and $10.1 \%$ more likely to have evidence of stamen bifurcation (likelihood ratio test $=30.43$ and likelihood ratio test $=16.94, p<0.001$ in both cases). Stamen primordia were initiated on the double buds at a bud radius of $244.1 \mu \mathrm{m}$ and stamen bifurcation at $378.4 \mu \mathrm{m}$ (Fig. 4). For every $1 \mu \mathrm{m}$ increase in bud radius, a double bud was $8.8 \%$ more likely to have stamen primordia and $3.4 \%$ more likely to have evidence of stamen 
primordia bifurcation (likelihood ratio test $=51.94$ and likelihood ratio test $=9.644, p<0.001$ and $p<0.01$ respectively).

\section{Allometry of Stamen Primordia}

For stamen primordia length vs. floral meristem radius, the equation describing the linear regression for the single phenotype had a value of 0.8192 and a value of 0.80267 for the double phenotype (Fig. 5). Both regressions showed a significant relationship between stamen primordia length and floral meristem radius (F-test, $p<0.001)$. The slope value for the single phenotype scaled more equally (was closer to 1.0) than the slope value for the double phenotype meaning that the single phenotype had a greater increase in stamen primordia length than the double phenotype over the same increase in floral meristem radius. This suggests that single stamen primordia are longer than double stamen primordia.

The linear regression for stamen primordia width vs. floral meristem radius did not show a significant relationship in the single phenotype (F-test, $p>0.04)$. There is no linear relationship between stamen primordia width and floral meristem radius suggesting that the width of stamen primordia was relatively constant in the single phenotype. However, the double phenotype did show a significant relationship between stamen primordia width and floral meristem radius (Ftest, $p<0.001$ ). The equation describing the relationship had a slope value of 0.6048 (Fig. 5). Unlike the single phenotype, this significant slope value suggests that the width of the double stamen primordia increased with the radius of the floral meristem. This suggests that the double stamen primordia were wider than the single stamen primordia.

Both the single and the double showed linear relationships between stamen primordia width and length (F-test, $p<0.001)$. The equation describing the linear regression of stamen primordia width vs. length had a slope value of 0.2865 for the single phenotype and a slope value 
of 0.5619 for the double phenotype (Fig.5). The slope value for the double phenotype is over two times the magnitude of the slope value of the single phenotype. This difference in the magnitude of slope values suggest that the double phenotype showed a much greater increase in width than did the single phenotype. A more equal scaling of stamen primordia width and length suggests that double stamen primordia were therefore more circular than single stamen primordia.

\section{Morphology and Size of Stamen Primordia}

During the frame of development examined here, the mean length of single stamen primordia was $125.8 \mu \mathrm{m}$, and the mean length of double stamen primordia was $113.8 \mu \mathrm{m}$. Single stamen primordia were significantly longer than double stamen primordia (permutation t-test, $p<0.001)$. With respect to the odds ratio from the logistic regression of phenotype vs. stamen primordia length, a stamen primordium was $1.2 \%$ more likely to be from a single bud than a double bud for every $1 \mu \mathrm{m}$ increase in stamen primordium length (likelihood ratio test $=11.39$, $p<0.001)$. Based on the inflection point from the logistic regression, a stamen primordium length of $130.1 \mu \mathrm{m}$ was more likely to be found on a single bud than on a double bud (Fig. 6).

During the same frame of development, the mean width of single stamen primordia was $83.55 \mu \mathrm{m}$, and the mean width of double stamen primordia was $86.62 \mu \mathrm{m}$. Double stamen primordia were significantly wider than single stamen primordia (permutation t-test, $p<0.05$ ). With respect to the odds ratio from the logistic regression of phenotype vs. stamen primordia width, a stamen primordium was $10.6 \%$ more likely to be from a double bud than a single bud for every $1 \mu \mathrm{m}$ increase in stamen primordium width (likelihood ratio test $=4.011, p<0.05$ ). According to the inflection point of the logistic regression, a stamen primordium width of $77.52 \mu \mathrm{m}$ was more likely to be found on a double bud than on a single bud (Fig. 7). 
The mean shape (i.e. length divided by width) of single stamen primordia was 1.504 , and the mean shape of double stamen primordia was 1.311. Single stamen primordia were significantly more elliptical than double stamen primordia (permutation t-test, $p<0.001$ ). With respect to the odds ratio from the logistic regression of phenotype vs. stamen primordium shape, a stamen primordium was 8.37 times more likely to be from a single bud than a double bud for every unit increase in stamen primordium shape (likelihood ratio test $=31.42, p<0.001$ ). The inflection point of the logistic regression suggest that a stamen primordium shape of 1.451 was more likely to be found on a single than on a double bud (Fig. 8).

The mean size of single stamen primordia was found to be $209.4 \mu \mathrm{m}$, and mean size of double stamen primordia was found to be $200.4 \mu \mathrm{m}$. The size of the single and double stamen primordia were determined not to be statistically different (permutation t-test, $p>0.05$ ). 


\section{Discussion}

Morphological differences in mature flowers correspond to developmental differences between the single and double phenotypes of $H$. rosa-sinensis. Our results show that the initiation of petal primordia occurred at the same bud radii in both phenotypes, but stamen primordia initiation and stamen primordia bifurcation occurred at significantly smaller bud radii in the singles than in the doubles. The allometry of the single phenotype is consistent with a transition from a circular to elliptic shape for the stamen primordia. The allometry of the double suggests a more circular shape throughout the frame of development considered here. With respect to stamen morphology, the stamen primordia of single phenotypes were longer, narrower, and therefore more elliptical than those of double phenotypes that were shorter, wider, and thus more circular. However, the size of the stamen primordia was not different between the two phenotypes during the frame of development considered here.

These results suggest that there are measurable differences between the two phenotypes at very early stages of floral development, namely with respect to organogenesis, allometry, and morphology of stamen primordia. There is also the obvious loss of symmetry and arrangement of stamen primordia in the double phenotype, and this loss of symmetry was also noted in previous studies (MacIntyre and Lacroix 1996). How do we combine the results from organogenesis, allometry, morphology, and size analyses, in addition to visual notes about symmetry and arrangement, to say something meaningful about the developmental and phenotypic differences between the two phenotypes, both during development and the consequences of this divergent development for mature flowers? Considering all of the findings together, it appears that the developmental differences between the two phenotypes can be linked to the double phenotype producing a larger floral meristem than the single phenotype after the initiation of the petal primordia on the ring of meristematic tissue. The difference between the two phenotypes with 
respect to the amount of available space on the floral meristem may explain the developmental differences noted in this study.

Organogenesis analyses showed that both phenotypes initiated petal primordia at approximately the same floral meristem radii (Fig. 4). After the initiation of petal primordia, the single and double phenotype begin to show developmental divergence with the double phenotype initiating stamen primordia and stamen primordia bifurcation at a larger meristem radius than the single phenotype (Fig. 4). The double phenotype initiated stamen primordia at a meristem radius of $244.1 \mu \mathrm{m}$ compared to meristem radius of $202.5 \mu \mathrm{m}$ in the single phenotype. This represents a difference of $41.6 \mu \mathrm{m}$ in the radius of the floral meristem between the two phenotypes. If the floral meristem is treated as a circle for the purpose of calculating surface area, that is almost a $45 \%$ increase in surface area at the point of stamen primordia initiation between the single and double phenotypes. At the point of stamen primordia bifurcation, the larger floral meristem of the double phenotype differed in surface between the two phenotypes by $56 \%$.

Considering space as a driver of development and morphology is a new area of interest in developmental botany (Prusinkiewicz and de Reuille 2010). Space-based models are being used to understand patterns of leaf venation (Runions et al. 2005), the emergence of fruit forms (Yin et al. 2008), and phyllotaxy (Hotton et al. 2006). Space is being considering with respect to floral development, too. The floral meristem, from which floral organ primordia are initiated, is now shown to be a space-limited structure that is determinate and lacks apical growth (ClaßenBockhoff 2015). An increase in the amount of space on the floral meristem is linked to the initiation of new organ primordia in some double flowers of Alcea rosea (Naghiloo et al. 2014), as well as a novel structure during the floral development of species in genus Passiflora (ClaßenBockhoff and Meyer 2016). Results presented here suggest that the concept of space can help us understand the divergent development of the single and double phenotypes of H. rosa-sinensis as 
it pertains to the available space on the ring of floral meristem during organogenesis of stamen primordia.

Results presented here suggest that producing a larger floral meristem has developmental consequences during stamen primordia initiation and development. According to accepted models, organ primordia are initiated at the further distance from neighbouring primordia (Reinhardt et al. 2005; Smith et al. 2006). There are a few proposed mechanisms to explain this phenomenon, but the most likely mechanism seems to be related to the formation of local auxin maxima (Reinhardt et al. 2003; Mourik et al. 2012). In the case of H. rosa-sinensis, this model would suggest that the stamen primordia of the double phenotype have more space between themselves than the stamen primordia of the single phenotype, as is supported by large differences in floral meristem radii reported in the organogenesis analyses. What are the consequences of this additional space for the development of stamen primordia in the double phenotype? Results of analyses suggest that additional space may be one factor that allows for (or is linked to) the manifestation of blended petal-anther structures that become increasingly obvious, and larger than normal stamen, as floral development progresses in the double phenotype. Other work on plant development has shown that plants maximize the use of meristem space during organ primordia initiation and development (Prusinkiewicz and Barbier de Reuille 2010).

This increased space on the floral meristem may also explain the breakdown of the positioning and symmetry of stamen primordia in the double phenotype. The single phenotype maintains stamen primordia arrangement throughout development, but the double phenotype does not. This difference was noted during this study, and also during previous work on $H$. rosasinensis (MacIntyre and Lacroix 1996). Considering the differences in space on the floral meristem, the breakdown of symmetry in the arrangement of stamen primordia of the double 
phenotype may also be a result of increased space on the floral meristem. Prusinkiewicz and de Reuille (2010) showed that marginal growth of structures that initiate organ primordia during plant development results in a loss of radial symmetry because the relatively flat disc of growing tissue is no longer flat, but instead loses its radial symmetry as it becomes increasingly more wave-like.

When comparing the mature flowers of the two phenotypes, the most striking difference between them is the presence of merged petal-anther structures (termed petaloids) that are part of the mature flowers of the double phenotype. These petaloids are considered to be an example of a homeotic mutation, or homeosis, where an organ occupies a position that is would not normally, but is not necessarily a 1:1 substitution (Sattler 1988; Lord et al. 1994). Results from this study suggest that homeotic mutations may lead to the establishment of different space constraints. In the case of the double phenotype of $H$. rosa-sinensis, a larger floral meristem may mean that the stamen primordia occupying this novel space appear to be subject to factors controlling the development of both petals and stamens, as signified by the merged petal-anther morphology of the petaloids. MacIntyre and Lacroix (1996) noted a gradient of petaloid morphology with some petaloids looking more like petals, and petaloids closer to stamen looking more like stamen. Although speculative, results from this study imply that the gradient of structures reflects the influence, or perhaps competition, of factors controlling petal and stamen development in a novel space on the floral meristem of the double phenotype. With respect to the ABC model, it seems that a gradient of expression of A and B (petals) and B and C (stamens) organ identity genes in the additional tissue of the floral meristem could account for the range of morphologies exhibited by the petaloids (Coen and Meyerowitz 1991; Theissen et al. 2000; Heijmans et al. 2012). 
But why does the double phenotype produce a larger floral meristem than the single phenotype after the initiation of floral primordia? While this is outside the scope of this study, other research suggests that hormones, such as auxin, gibberellin, and cytokinin, are functionally linked A, B and C class organ identity genes during floral development, and these hormones are also responsible for controlling the size of the floral meristem and the initiation of organ primordia (Gómez-Mena at al. 2005; Kaufmann et al. 2009; Kaufmann et al. 2010; Chandler 2011). A and B class genes control petal development, and B and C class genes control stamen development. If there are in fact differences with respect to organ identity genes between the two phenotypes, mutations in organ identity genes would explain the divergent morphology between the two phenotypes, in addition to explain the difference in the size of the floral meristem between the single and double phenotype (Coen and Meyerowitz 1991; Chandler 2011). The relationship between hormones, floral development, and floral meristem size is an area of research that obviously warrants further investigation. 


\section{Acknowledgements}

This research was supported by the Natural Sciences and Engineering Research Council (NSERC) of Canada in the form of three Undergraduate Student Research Awards (USRAs) to CHN. More broadly, the resources needed to complete this study were funded by a NSERC Discovery Grant to CRL. 


\section{References}

Ai, Y., Zhang, C., Sun, Y., Wang, W., He, Y., and Bao, M. 2017. Characterization and Functional Analysis of Five MADS-Box B Class Genes Related to Floral Organ Identification in Tagetes erecta. Plos One, 12: 1. doi.org/10.1371/journal.pone.0169777.

Behrend, A., Borchert, T., and Hohe, A. 2015. "The usual suspects"- analysis of transcriptome sequences reveals deviating B gene activity in C. vulgaris bud bloomers. BMC Plant Biol. 15: 8. doi.org/10.1186/s12870-014-0407-z.

Belyaeva, R.G. 1995. Genetic study of double flower mutation in plants with monocarpic shoot. Genetika, 31: 674-677.

Causier, B., Schwarz-Sommer, Z., and Davies, B. 2010. Floral organ identity: 20 years of ABCs. Semin. Cell Dev. Biol. 21: 73-79.

Chandler, J. W. 2011. The Hormonal Regulation of Flower Development. J. Plant Growth Regul. 30(2): 242-254. doi.org/10.1007/s00344-010-9180-x.

Claßen-Bockhoff, R. 2015. The shoot concept of the flower: Still up to date?. Flora, 221: 46-53. doi.org/10.1016/j.flora.2015.11.012.

Claßen-Bockhoff, R. and Meyer, C. 2016. Space matters: Meristem expansion triggers corona formation in Passiflora. Ann. Bot-London, 117(2): 277-290. doi.org/10.1093/aob/mcv177

Coen, E.S. and Meyerowitz, E.M. 1991. The war of the whorls: genetic interactions controlling flower development. Nature, 353(6339): 31-37.

Cohen, J., Litt, A., and Davis, J.I. 2012. Comparative floral development in Lithospermum (Boraginaceae) and implications for the evolution and development of heterostyly. Ann. Bot. 99(5): 797-805.

Dubois, A., Raymond, O., Maene, M., Baudino, S., Langlade, N.B., Boltz, V., Vergne, P. and Bendahmane, M. 2010. Tinkering with the C-function: a molecular frame for the selection 
of double flowers in cultivated roses. Plos One, 5(2):e9288.

doi.org/10.1371/journal.pone.0009288.

Firth, D. 1993. Bias Reduction of Maximum Likelihood Estimates. Biometrika, 80(1): 27-38.

Freyre, R., Moseley, A., Wilson, S.B., and Knox, G.W. 2012. Breeding and evaluating for landscape performance and fruitlessness in Mexican petunia (Ruellia, Acanthaceae). Hortscience, 47 (9): 1245-1251.

Galimba, K.D., Tolkin, T.R., Sullivan, A.M., Melzer, R., Theissen, G., and Di Stilio, V.S. 2012. Loss of deeply conserved C-class floral homeotic gene function and C- and E-class protein interaction in a double-flowered ranunculid mutant. Proc. Natl. Acad. Sci. U. S. A. 109(34): E2267-E2275.

Gomez-Mena, C., de Folter, S., Costa, M.M., Angenent, G.C., and Sablowski, R. 2005. Transcriptional program controlled by the floral homeotic gene AGAMOUS during early organogenesis. Development, 132(3): 429-438.

Heijmans, K., Morel, P., and Vanderbussche, M. 2012. MADS-box genes and floral development: the dark side. J. Exp. Bot. 63(15): 5397-5404.

Heinze, G. and Schemper, M. 2002. A solution to the problem of separation in logistic regression. Stat. Med. 21: 2409-2419.

Honma, T. and Goto, K. 2001. Complexes of MADS-box proteins are sufficient to convert leaves into floral organs. Nature, 409(6819): 525-529.

Hotton, S., Johnson V., Wilbarger, J., Zwieniecki, K., Atela, P., Golé, C., and Dumais, J. 2006. The possible and the actual in phyllotaxis: bridging the gap between empirical observations and iterative models. J. Plant Growth Regul. 25(4): 313-323.

Innes, R., Remphrey, W., and Lenz, L. 1989. An analysis of the development of single and double flowers in Potentilla fruticosa. Can. J. Bot. 67(4): 1071-1079. 
Kapoor, M., Baba, A., Kubo, K., Shibuya, K., Matsui, K., Tanaka, Y., and Takatsuji, H. 2005. Transgene-triggered, epigenetically regulated ectopic expression of a flower homeotic gene pMADS3 in Petunia. The Plant Journal, 43(5): 649-661. https://doi.org/10.1111/j.1365-313X.2005.02481.

Kaufmann, K., Muiño, J.M., Jauregui, R., Airoldi, C.A., Smaczniak, C., Krajewski, P., and Angenent, G. 2009. Target genes of the MADS transcription factor SEPALLATA3: integration of developmental and hormonal pathways in the Arabidopsis flower. Plos Biol. 7(4): e1000090. doi.org/10.1371/journal.pbio.1000090.

Kaufmann, K., Wellmer, F., Muiño, J.M., Ferrier, T., Wuest, S.E., Kumar, V., Serrano-Mislata, A., Madueño, F., Krajewski, P., Meyerowitz, E.M., Angenent, G.C., and Riechmann, J.L. 2010. Orchestration of floral initiation by APETALA1. Science, 328(5974): 85-89.

Liu, C., He, Y., Gou, T., Li, X., Ning, G., and Bao, M. 2016. Identification of molecular markers associated with the double flower trait in Petunia hybrida. Sci. Hortic-Amsterdam, 206: 43-50.

Lord, E.M., Crone, W., and Hill, J.P. 1994. Timing of events during flower organogenesis: Arabidopsis as a model system. Curr. Top. Dev. Biol. 29: 325-356.

MacIntyre, J. P., Lacroix, C. R. 1996. Comparative development of perianth and androecial primordia of the single flower and the homeotic double-flowered mutant in Hibiscus rosasinensis (Malvaceae). Can. J. Bot. 74: 1871-1882.

McLellan, T. 1990. Development of differences in leaf shape in Begonia dregei (Begoniaceae). Am. J. Bot. 77(3): 323-337.

Naghiloo, S., and Classen-Bockhoff, R. 2016. Developmental analysis of merosity and sexual morphs in Rubiaceae: A case study in Rubia and Cruciata. Flora, 222: 52-59.

Naghiloo, S., Esmaillou, Z., and Dadpour, M.R. 2014. Comparative floral ontogeny of single- 
flowered and double-flowered phenotypes of Alcea rosea (Malvaceae). Aust. J. Bot. 62 (3): 217-228.

Nakatsuka, T., Saito, M., Yamada, E., Fujita, K., Yamagishi, N., and Yoshikawa, N. 2015. Isolation and characterization of the C-class MADS-box gene involved in the formation of double flowers in Japanese gentian. BMC Plant Biol. 1: 14. doi.org/10.1186/s12870-015-0569-3.

Prusinkiewicz, P., and Barbier De Reuille, P. 2010. Constraints of space in plant development. J. Exp. Bot. 61(8): 2117-2129. doi.org/10.1093/jxb/erq081.

Reinhardt, D., Frenz, M., Mandel, T., and Kuhlemeier, C. 2005. Microsurgical and laser ablation analysis of leaf positioning and dorsoventral patterning in tomato. Development, 132(1): $15-26$.

Reinhardt, D., Pesce, E. R., Stieger, P., Mandel, T., Baltensperger, K., Bennett, M., Traas, J., Friml, J., and Kuhlemeier, C. 2003. Regulation of phyllotaxis by polar auxin transport. Nature, 426: 255-260.

Roberston, M. 1984. The effect of environmental factors on flower color and petal number of Potentilla fruticosa L. M.Sc. thesis. University of Manitoba, Winnipeg.

Runions, A., Fuhrer, M., Lane, B., Federl, P., Rolland-Lagan, A.G., and Prusinkiewicz, P. 2005. Modeling and visualization of leaf venation patterns. ACM Transactions on Graphics (TOG), 24(3): 702-711.

Sattler, R. 1988. Homeosis in Plants. Am. J. Bot. 75(10): 1606-1617.

Scovel, G., Ben-Meir, H., Ovadis, M., Itzhaki, H., and Vainstein, A. 1998. RAPD and RFLP markers tightly linked to the locus controlling carnation (Dianthus caryophyllus) flower type. Theor. Appl. Genet. 96: 117-122.

Smaczniak, C., Immink, R.G., Muiño, J.M., Blanvillain, R., Busscher, M., Busscher-Lange, J., 
Dinh, Q.P., Liu, S., Westphal, A.H., Boeren, S. and Parcy, F. 2012. Characterization of MADS-domain transcription factor complexes in Arabidopsis flower development. Proc. Natl. Acad. Sci. U.S.A. 109(5): 1560-1565.

Smith, R.S., Kuhlemeier, C., and Prusinkiewics, P. 2006. Inhibition fields for phyllotactic pattern formation: a simulation study. Can. J. Bot. 84(11): 1635-1649.

Sun, Y., Fan, Z., Li, X., Liu, Z., Li, J., and Yin, H. 2014. Distinct double flower varieties in Camellia japonica exhibit both expansion and contraction of C-class gene expression. BMC Plant Biol. 14: 288. doi.org/10.1186/s12870-014-0288-1.

Theissen, G., Becker, A., Di Rosa, A., Kanno, A, Kim, J.T., Münster, T., Winter, K.U., and Saedler, H. 2000. A short history of MADS-box genes in plants. Plant Mol. Biol. 42: $115-149$.

Theissen, G., Melzer, R., and Rümpler, F. 2016. MADS-domain transcription factors and the floral quartet model of flower development: linking plant development and evolution. Development, 143: 3259-3271.

van Mourik, S., Kaufmann, K., van Dijk, A.D.J., Angenent, G. C., and Roeland, M. H. 2012. Simulation of Organ Patterning on the Floral Meristem Using a Polar Auxin Transport Model. Plos One, 7(1): 1-9. doi.org/10.1371/journal.pone.0028762.

Yanofsky, M. and Schmidt, R. J. 1999. MADS-box genes and plant development. Trends Plant Sci. 4: 1360-1385.

Yin, J., Cao, Z., Li, C., Sheinman, I., and Chen, X. 2008. Stress-driven buckling patterns in spheroidal core/shell structures. Proc. Natl. Acad. Sci. U.S.A. 105(49): 19132-19135.

Zainol, R., Stimart, D.P., and Evert, R.F. 1998. Anatomical analysis of double flower morphogenesis in a Nicotiana alata mutant. J. Am. Soc. Hort. Sci. 123: 967-972. 


\section{Figure Legends}

\section{Figure 1}

Images of mature flowers of the single phenotype (A) and double phenotype (B), as well as SEM images of a single bud (C) and double bud (D) that were outside the specific developmental range considered for this study. Mature flowers are 7.5 to $9 \mathrm{~cm}$ in diameter. White bars at the bottom right of images $C$ and $D$ represent $400 \mu \mathrm{m}$. In image $C$, note the uniform appearance of developing structures. In image $\mathrm{D}$, note the lack of uniformity concerning the shape and size of developing structures.

\section{Figure 2}

Developmental series for single phenotype. Series reads left to right, top to bottom. $\mathrm{R}_{\mathrm{av}}$ represents the radius of the meristematic ring based on the average of three radial measurements. Image A represents the earliest stage of development of the floral meristem (meristematic ring) used in the time frame of this study, and image $\mathrm{H}$ represents the latest stage of development. Note the initiation of petal primordia (PP) in image $\mathrm{B}$, stamen primordia (SP) in image $\mathrm{C}$, and bifurcation of stamen primordia (SPB) in images $\mathrm{G}$ and $\mathrm{H}$.

\section{Figure 3}

Developmental series for double phenotype. Series reads left to right, top to bottom. $\mathrm{R}_{\mathrm{av}}$ represents the radius of the meristematic ring based on an average of three radial measurements. Image A represents the earliest stage of development of the floral meristem (meristematic ring) used in the time frame of this study, and image $\mathrm{H}$ represents the latest stage of development. Note initiation of petal primordia (PP) in image $\mathrm{B}$, stamen primordia (SP) in image $\mathrm{D}$, and bifurcation of stamen primordia (SPB) in image $\mathrm{H}$. 


\section{Figure 4}

Inflection points for the initiation of petal primordia, stamen primordia, and stamen primordia bifurcation for the single and double phenotypes estimated via logistic regression using Firth's penalized-likelihood method. The inflection point represents the floral meristem radius (i.e. bud radius) after which a structure was more likely to be present than not present. Whiskers represent the confidence intervals predicted by bootstrapping. The petal primordia were initiated at bud radii in the single and double phenotype that were not statistically different, and this nonsignificant difference is designated with an n.s. in the figure. The stamen primordia of the single phenotype were initiated at a significantly smaller bud radius than the double phenotype. Stamen bifurcation in the single phenotype was also initiated at statistically smaller bud radii than stamen bifurcation in the double phenotype. The statistically significant differences between the single and double phenotypes concerning the bud radii at which stamen primordia and stamen primordia bifurcation were initiated is noted by sig. in the figure.

\section{Figure 5}

Summary of allometric analyses for (A) Stamen primordia length vs. floral meristem radius (B) Stamen primordia width vs. floral meristem radius and (C) stamen primordia width vs. stamen primordia length. The y-axis represents the allometric relationships between morphological measurements, which are defined as the values of the slopes from the linear regressions on the logged measurements. The slope value is less than one in all cases indicating that length, width, and shape of stamen primordia grew less rapidly than floral meristem radius in all cases for both the single and double phenotype. Only the slope of the width of stamen primordia vs. floral meristem radius analysis for the single phenotype was non-significant (F-test, $p>0.05$, denoted 
by n.s.). The other slope values were all significant (F-test, $p<0.001$, denoted by sig.). The length of single stamen primordia scaled more equally with floral meristem radius than did the length of the double stamen primordia suggesting that single stamen primordia were longer than double stamen primordia. The width of the double stamen primordia scaled more equally with floral meristem radius than did the width of single stamen primordia suggesting that double stamen primordia were wider than single stamen primordia. The width and length of the double stamen primordia scaled more equally than the width and length of single stamen primordia, therefore suggesting that double stamen primordia were more circular than single stamen primordia.

\section{Figure 6}

Plot of the lengths of single (black dots) and double (grey dots) stamen primordia across floral meristem radii. The middle line is the inflection point calculated using a logistic regression model. The inflection point is a horizontal line because the inflection point is a fixed value. After the inflection point of $130.1 \mu \mathrm{m}$, a length measurement was more likely to be from the single phenotype than the double phenotype (likelihood ratio test $=11.39, p<0.001$ ), suggesting that single stamen primordia are longer than double stamen primordia. The upper and lower lines represent bootstrapped $95 \%$ confidence internals around the inflection point.

\section{Figure 7}

Plot of the widths of single (black dots) and double (grey dots) stamen primordia across floral meristem radii. The middle line is the inflection point calculated using a logistic regression model. The inflection point is a horizontal line because the inflection point is a fixed value. After the inflection point of $77.52 \mu \mathrm{m}$, a width measurement was more likely to be from the double 
phenotype than the single phenotype (likelihood ratio test $=4.011, p<0.05$ ), suggesting that double stamen primordia are wider than single stamen primordia. The upper and lower lines represent bootstrapped $95 \%$ confidence internals around the inflection point.

\section{Figure 8}

Plot of the length/width (shape) of single (black dots) and double (grey dots) stamen primordia across floral meristem radii. The middle line is the inflection point calculated using a logistic regression model. The inflection point is a horizontal line because the inflection point is a fixed value. After the inflection point of 1.451 , a length/width ratio was more likely to be from the single phenotype than the double phenotype (likelihood ratio test $=31.42, p<0.001$ ), suggesting that single stamen primordia are more elliptic and double stamen primordia are more circular. The upper and lower lines represent bootstrapped 95\% confidence internals around the inflection point. 

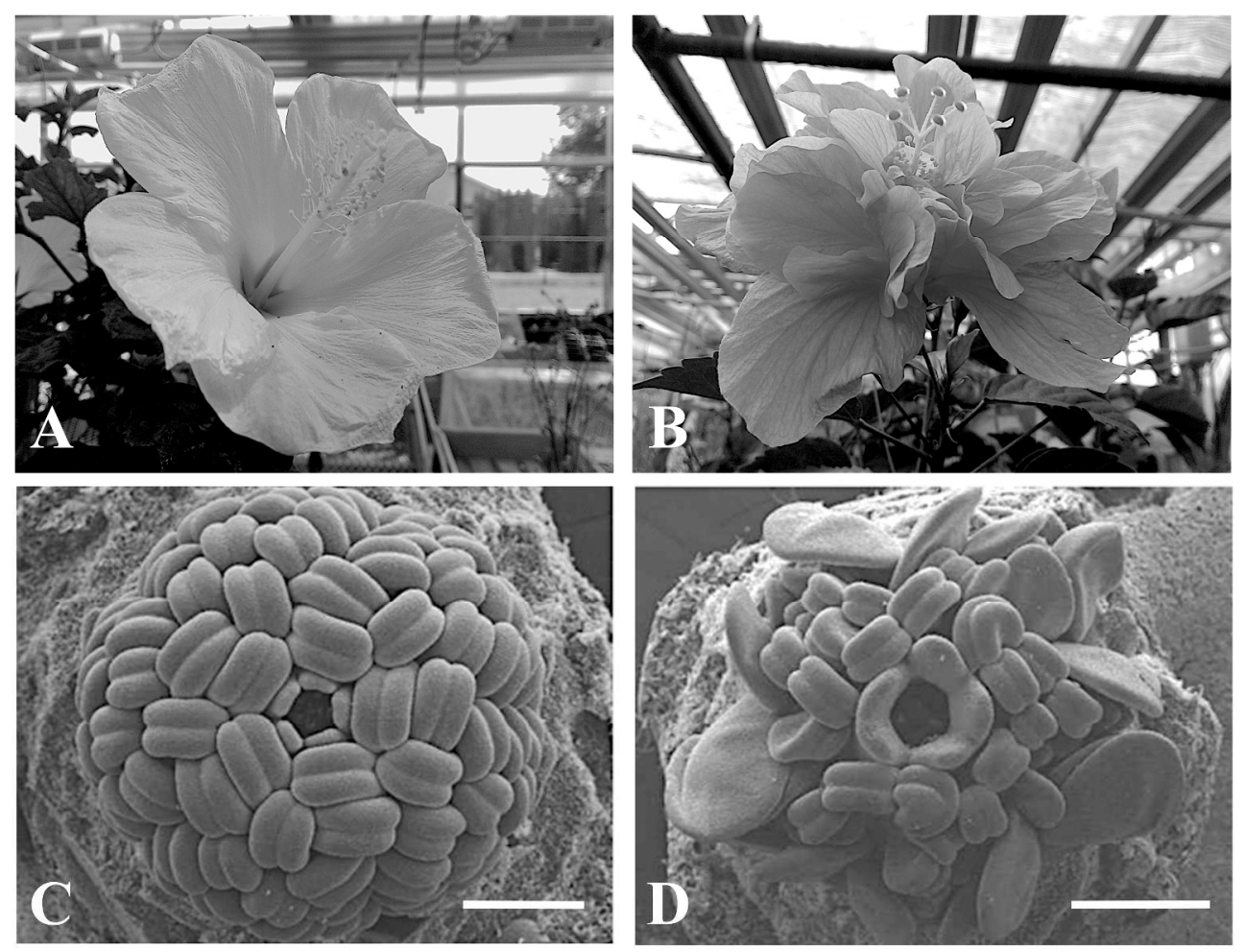

figure 1

$191 \times 148 \mathrm{~mm}(600 \times 600$ DPI $)$ 



figure 2

$282 \times 426 \mathrm{~mm}(600 \times 600 \mathrm{DPI})$ 

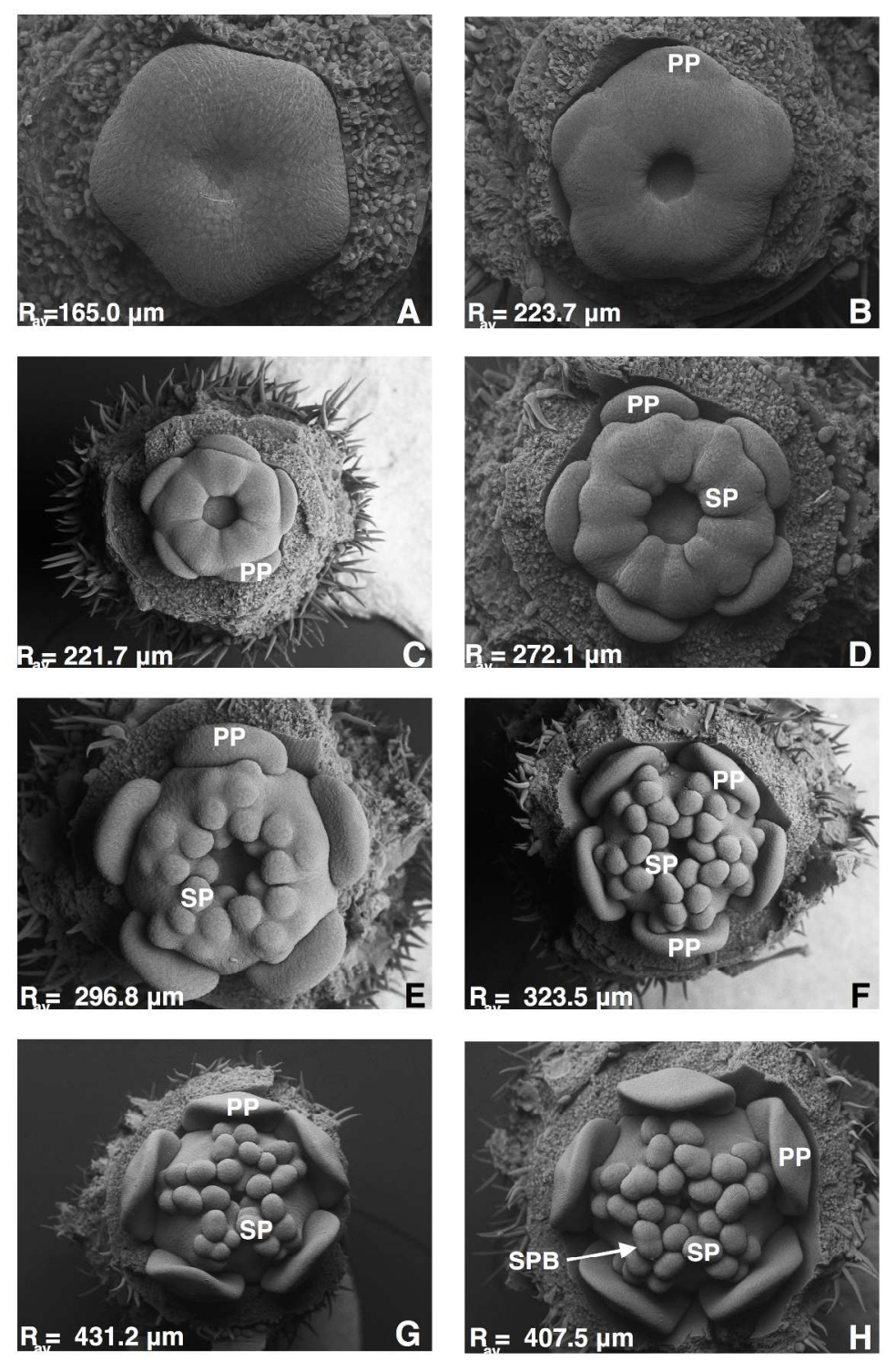

figure 3

$292 \times 430 \mathrm{~mm}(300 \times 300 \mathrm{DPI})$ 


\section{Summary of Organogenesis Analyses}

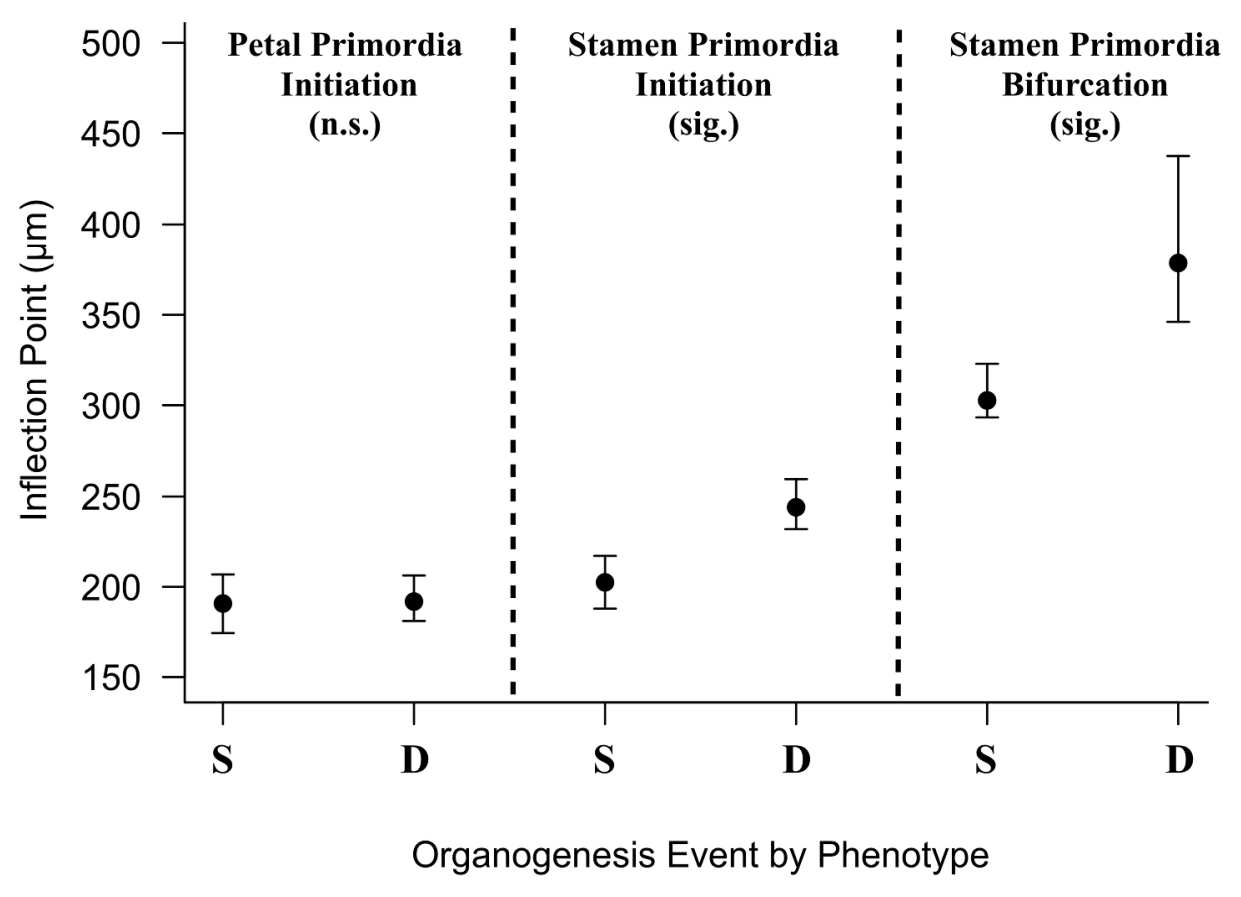

figure 4

$127 \times 105 \mathrm{~mm}(600 \times 600 \mathrm{DPI})$ 


\section{Summary of Allometric Analyses}

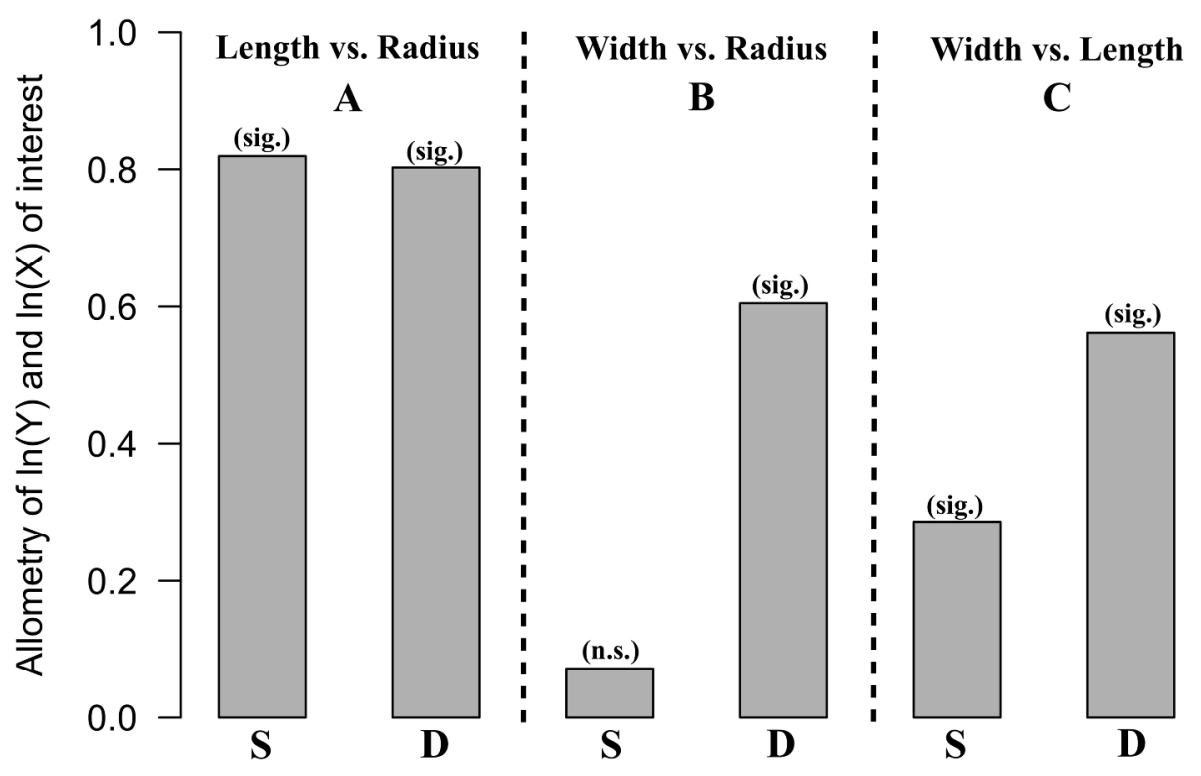

Allometric Comparison by Phenotype

figure 5

$127 \times 105 \mathrm{~mm}(600 \times 600 \mathrm{DPI})$ 


\section{Inflection Point for Stamen Primordial Length (95\% Cl)}



figure 6

$127 \times 105 \mathrm{~mm}(600 \times 600 \mathrm{DPI})$ 


\section{Inflection Point for Stamen Primordial Width $(95 \% \mathrm{Cl})$}



figure 7

$127 \times 105 \mathrm{~mm}(600 \times 600 \mathrm{DPI})$ 


\section{Inflection Point for Stamen Primordial Shape (95\% Cl)}

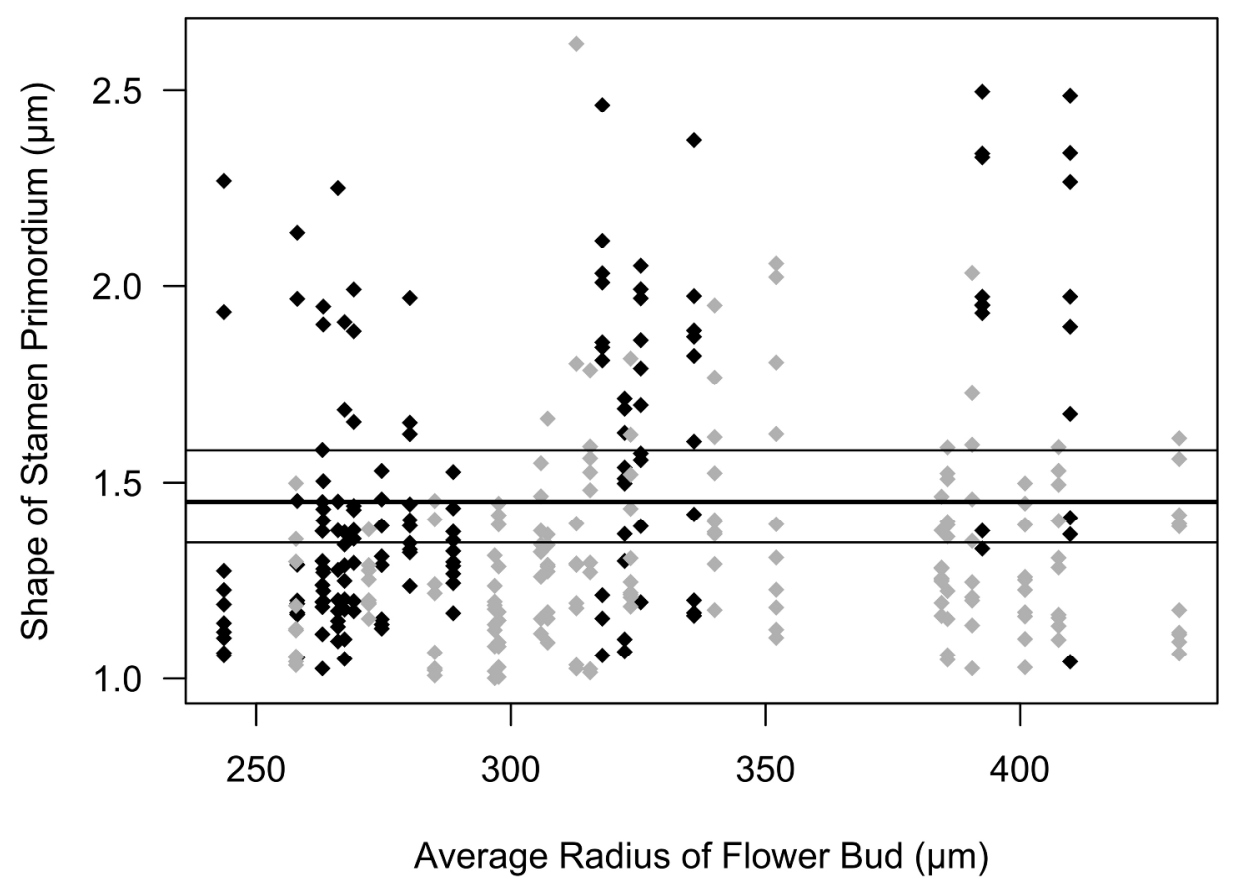

figure 8

$127 \times 105 \mathrm{~mm}(600 \times 600 \mathrm{DPI})$ 